\title{
A new class of monohedral pentagonal spherical tilings with GeoGebra
}

\author{
Ana M. D’Azevedo Breda and José M. Dos Santos Dos Santos
}

(Communicated by João Araújo and Peter J. Cameron)

\begin{abstract}
By a monohedral spherical tiling we mean a decomposition of the sphere by geodesic congruent polygons. Here, making use of GeoGebra, a well known free interactive mathematics software, we show how to generate new classes of monohedral non-convex triangular and new non-convex pentagonal spherical tilings, changing the side gluing rules of the regular spherical tetrahedral tiling, by means of a local action of particular subgroups of spherical isometries. In both cases each face has $\pi$ as area measure.

In relation to the new class of pentagonal tilings, we describe some of their properties and show the existence, in a special case, of an associated dihedral triangular spherical tiling, that is, a tiling composed by two sets of congruent triangles.

These classes of spherical tilings have emerged as a result of an interative construction process, only possible by the use of newly produced GeoGebra tools and the dynamic interaction capabilities of this software.
\end{abstract}

Mathematics Subject Classification: 51L99, 58E40

Keywords: Spherical geometry, spherical tilings, GeoGebra

\section{Introduction}

In this paper our main result is the description of the combinatorial and geometric characterisation of a new one-parameter class of edge-to-edge spherical tilings, denoted by $\mathfrak{B}_{\{\mathcal{C}, \rho] \rho \in[0, \pi]}$, expanding the knowledge of monohedral spherical tilings by triangles and pentagons, that is, tilings of the sphere in which all spherical faces are congruent among them. This one-parameter class emerged as a result of an iterative construction process, starting from a particular subset of $S^{2}$ and particular sets of spherical isometries ruling the gluing side rules of the new constructed tilings (for details, see Section 4), making use of new produced GeoGebra tools and the dynamic interaction capabilities of this software. 
There are many tools to work with spherical geometry in an interactive way, as Sphaerica [8], Spherical Easel [1], and Povray [5]. However, for our purposes we need to work with more flexible tools and commands, in particular, we need to obtain in real time the orbit of a set of spherical points under the action of a (sub) group of spherical isometries. For that, GeoGebra [10] seems the best option for two crucial reasons: the widespread use of GeoGebra and the possibility of interaction with geometrical and algebraic representations simultaneously. In fact, GeoGebra has several geometrical representations in 2 and 3 dimensions allowing the interaction with spherical points in a diversity of ways. Besides, the algebraic capabilities of GeoGebra allow the study and the induction of some geometrical properties which may be visualized in real time. Among its many features, GeoGebra allows the creation of new tools and commands, dealing with sequences of various geometric and algebraic objects and using logical and heuristic procedures, it allows, to certify some properties of these same objects, for example, to be congruent with each other. [7].

A systematic study of spherical tilings started with D. Sommerville [11] who has stablished part of the classification of spherical tilings by isosceles triangles having analysed a very particular case by scalene triangles [6], p. 467. H. Davies, in 1967, presents an incomplete classification of triangular monohedral tilings of the sphere [4] omitting many details which were fixed latter on.

Tilings of the sphere by right triangles were obtained by Yukako Ueno and Yoshio Agaoka in 1996 [14]. Later, in 2002, the same authors [15] obtain the complete classification of monohedral edge-to-edge triangular spherical tilings. It should be noted that triangular spherical folding tilings were studied by Ana Breda [2] and their classification was obtained in 1992, these being a subset of the triangular monohedral spherical tilings .

The regular dodecahedral spherical tiling is a well known tiling of the sphere by twelve regular pentagonal spherical polygons. More recently, all edge-to-edge tilings of the sphere by 12 congruent convex pentagons it has been classified by Honghao, Shi and Yan [9].

The classification of spherical tilings by triangles is not yet completed. In fact, little is known when the condition of being monohedral or edge-to-edge is dropped out. A systematic study to enumerate and classify all spherical tilings is far from being complete.

In the next Section 2, we begin by presenting a construction process of monohedral spherical tilings of area $\pi$, this process depends on a spherical set locally under the action of a subgroup of spherical isometries. We will end up with two classes. In Section 3 we will describe the immersion of the class, $\mathfrak{I}_{(\mathcal{C}, p)}$, of monohedral spherical tilings by four triangles, followed, in Section 4, by the description of the finding of $\mathfrak{B}_{(\mathcal{C}, \rho)}$, a class of monohedral spherical tiling by nonconvex pentagons of area $\pi$. Finally, in Section 5, we present our conclusions 
about the use of GeoGebra in the present work, as well as our proposals for upcoming research.

From now on, all the tilings in consideration are edge-to-edge, unless stated otherwise.

\section{A class of monohedral tilings of the sphere of area $\pi$}

Let $S^{2}$ be the sphere centred in $O=(0,0,0)$ and radius $1, c$ a great circle of $S^{2}$, and $A$ and $B$ two distinct points in $c$ such that $\widehat{A O B}=\arcsin \left(\frac{1}{3}\right)+\frac{1}{2} \pi$. Chose one point $C \in S^{2}$ such that $[A B C]$ defines an equilateral triangle with angles $\frac{2 \pi}{3}$. Let $Q, R$ and $S$ be the midpoints of the spherical segments $\overparen{A B}, \overparen{B C}$ and $\overparen{C A}$, respectively. Let $P \in \overparen{Q C}$ such that $\widehat{Q O P}=\rho, \rho \in[0, \pi]$. Let $\mathcal{C}=\left\{X \in S^{2}: X \in \overparen{P S} \vee X\right.$ $\in \overparen{P R} \vee X \in \overparen{P Q}\}$. In order to obtain a spherical tiling, we use GeoGebra, applying spherical isometries to the set $\mathcal{C}$ (Fig. 1(a)). All the isometries that will be applied to $\mathcal{C}$ fix the points $A, B, C, Q, S, R$. In the case illustrated in Figure 1(b), only the point $P$ will be a vertex of the tiling and the points $Q$ and $R$ will be midpoints of edges of the tiling. In case of Figure 1(c), the points $Q, R$ will be vertices of the tiling and $S$ will be the midpoint of an edge of the tiling. Since the points $Q$, $P, R$ are midpoints of the spherical equilateral triangle $A B C$ of angles $\frac{2 \pi}{3}$, we have:

$$
\begin{gathered}
\widehat{Q S}=\overparen{S R}=\overparen{R Q}=\frac{\pi}{2}, \quad \widehat{P S}=\overparen{P R} \\
\widehat{A B C}=\widehat{B C A}=\widehat{C A B}=\frac{2 \pi}{3}, \quad \widehat{B S Q}=\widehat{S Q B}=\widehat{Q R A}=\widehat{A Q R}=\frac{\pi}{4}
\end{gathered}
$$

The lengths of the $\arcsin \mathcal{C}$ (arcs emerging from $P$ ) and the angles around $P$ are defined in function of $\rho$, using the spherical relations for triangles. Accordingly,
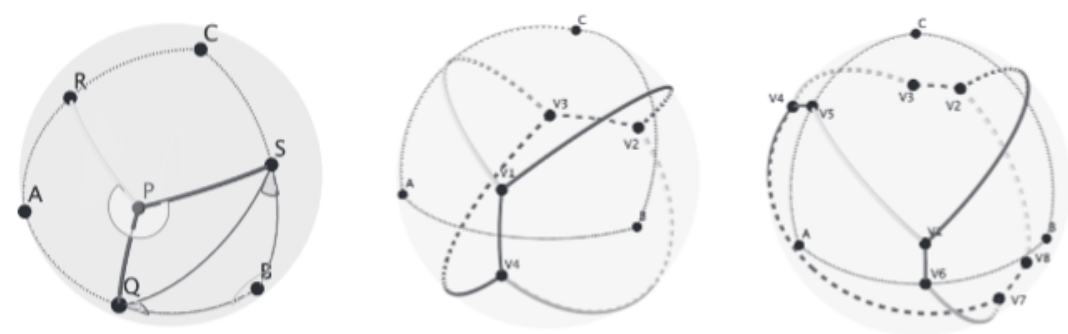

Figure 1. (a) Representation of the set $\mathcal{C}$. (b) monohedral triangular spherical tiling, $\mathfrak{I}_{(\mathcal{C}, \pi / 10)}$. (c) monohedral pentagons spherical tiling, $\mathfrak{P}_{(\mathcal{C}, \pi / 10)}$. 
we have:

$$
\begin{gathered}
\overparen{P Q}=\rho, \quad \overparen{P R}=\overparen{P S}=\arccos \left(\frac{\sqrt{2}}{2} \sin (\rho)\right) \\
\widehat{Q P S}=\widehat{Q P R}=\arccos \left(\frac{-\cos (\rho)}{\sqrt{1+\cos (\rho)^{2}}}\right) \\
\widehat{P R A}=\widehat{P S B}=\frac{\pi}{4}+\arccos \left(\frac{\sqrt{2} \cos (\rho)}{\sqrt{1+\cos (\rho)^{2}}}\right) .
\end{gathered}
$$

In order to obtain the two classes of monohedral spherical tilings, $\mathfrak{I}_{\{\mathcal{C}, \rho)}$ and $\mathfrak{B}_{\{\mathcal{C}, \rho)}$, respectively by triangles and pentagons, see Figure 1, we consider the rotations $\mathscr{R}_{(P, \rho)}$, about the axis $O P$, of angle $\rho, \rho \in[0, \pi]$, and two sets of spherical isometries $\Im_{1}$ and $\Im_{2}$ defined bellow.

Let be $\Im_{1}=\left\{\mathscr{R}_{(Q, \pi)}, \mathscr{R}_{(S, \pi)}, \mathscr{R}_{(R, \pi)}\right\}$ and $\Im_{2}=\left\{\mathscr{R}_{(S, \pi)}, \mathscr{R}_{(C, 2 \pi / 3)}\right\}$.

For each value of $\rho \in[0, \pi]$, the action of $\Im_{1}$ on $\mathcal{C}$ defines a class of spherical monohedral triangular tilings denoted by $\mathfrak{I}_{(\mathcal{C}, \rho)}$.

On the other hand, for each value of $\rho \in[0, \pi]$, we may construct a new class of monohedral tilings by non-convex pentagons denoted by $\mathfrak{B}_{(\mathcal{C}, \rho)}$.

In this case the four tiles of $\mathfrak{B}_{(\mathcal{C}, \rho)}$ are obtained using $\Im_{2}$ and applying the procedure indicated bellow, see Figure 1 (c).

Let

1. $\mathcal{C}_{0}=\mathcal{C}$

2. $\mathcal{C}_{1}=\mathscr{R}_{(S, \pi)}(\mathcal{C})$;

3. $\mathcal{C}_{2}=\mathscr{R}_{(\mathcal{C}, 2 \pi / 3)}\left(\mathcal{C}_{1}\right)$;

4. $\mathcal{C}_{3}=\mathscr{R}_{(S, \pi)}\left(\mathcal{C}_{2}\right)$.

Then, $\mathfrak{P}_{(\mathcal{C}, \rho)}=\bigcup_{i=0}^{3} \mathcal{C}_{i}$.

Let us see how GeoGebra had been used to generate the class of tilings $\mathfrak{I}_{(\mathcal{C}, \theta)}$, $\theta \in[0,2 \pi]$ and acted as support for some of the results presented here.

The first geometric construction was done starting from a point $P$ in $\overparen{Q C}$ and joinning $P$ to the middle points of $\overparen{A C}$ and $\overparen{B C}$, giving rise to $\mathcal{C}$. Applying to $\mathcal{C}$ each one of the isometries in $\Im_{1}$, a spherical configuration emerges.

The code used for visualizing, for each value of $\theta$, this configuration is shown in Table 1 .

If the obtained configuration is a spherical tiling, the CAS view is then used to obtain the algebraic expressions of the measures of: the arcs lengths; the angles surrounding each vertex and the coordinates of the vertices. Note that in the GeoGebra CAS view we do have all the vector and matrix operations needed to 


\begin{tabular}{|c|c|c|}
\hline Objects & $3 \mathrm{D}$ View & CAS View \\
\hline Parameter & $\theta-\operatorname{Slider}\left(0,2^{*} \mathrm{pi}, 2^{*} \mathrm{pi} / 100\right)$ & \\
\hline Points & $\begin{array}{l}C-(-1 / 3,-\operatorname{sqrt}(2 / 9), \operatorname{sqrt}(2 / 3)) \\
\mathrm{Q}-(\operatorname{sqrt}(3) / 3, \operatorname{sqrt}(2 / 3), 0) \\
\mathrm{S}-((-\operatorname{sqrt}(3)) / 3, \operatorname{sqrt}(2 / 3) / 2, \operatorname{sqrt}(2) / 2) \\
\mathrm{R}-(\operatorname{sqrt}(3) / 3,(-\operatorname{sqrt}(2 / 3)) / 2, \operatorname{sqrt}(2) / 2) \\
\mathrm{P}-\left(\cos (\theta)^{*} \operatorname{sqrt}(3) / 3, \cos (\theta){ }^{*} \operatorname{sqrt}(2 / 3), \sin (\theta)\right)\end{array}$ & $\begin{array}{l}\text { Correspond one vector to each wertex } \\
\mathrm{v} C:-C \\
\mathrm{v} Q:-Q \\
\mathrm{vS}:-\mathrm{S} \\
\mathrm{vR}:-\mathrm{R} \\
\mathrm{vP}:-\left(\cos (\rho)^{*} \operatorname{sqrt}(3) / 3, \cos (\rho)^{*} \operatorname{sqrt}(2 / 3), \sin (\rho)\right)\end{array}$ \\
\hline Ares & $\begin{array}{l}P Q-\operatorname{CircularArc}((0,0,0), P, Q, P l a n e((0,0,0), P, Q)) \\
P S-\operatorname{CircularArc}((0,0,0), P, S, P l a n e((0,0,0), P, S)) \\
P Q-\operatorname{CircularArc}((0,0,0), P, R, P l a n e((0,0,0), P, R))\end{array}$ & $\begin{array}{l}\arccos \left(\mathrm{v} P^{*} \mathrm{vQ}\right) \\
\arccos \left(\mathrm{vP}^{*} \mathrm{vS}\right) \\
\arccos \left(\mathrm{v} P^{*} \mathrm{vR}\right)\end{array}$ \\
\hline Cell & $\mathrm{Ce}-\{\mathrm{PQ}, \mathrm{PS}, \mathrm{PR}\}$ & \\
\hline $\mathscr{R}_{(Q, \pi)}$ & $\operatorname{IlCel}-\operatorname{Rotate}(\mathrm{Ce}, \mathrm{pi}, \operatorname{Ray}((0,0,0), Q))$ & \\
\hline $\mathscr{R}_{(\mathcal{S}, \pi)}$ & $x^{2}$ & $\begin{array}{l}\text { For example, defining the rotation matrix, } \\
\text { MSpi :- }\left(\begin{array}{ccc}-\frac{1}{3} & -\frac{\sqrt{2}}{3} & -\frac{\sqrt{6}}{3} \\
-\frac{\sqrt{2}}{3} & -\frac{2}{3} & 0 \\
-\frac{\sqrt{6}}{3} & \frac{1}{\sqrt{3}} & 0\end{array}\right) \\
\text { applying the vector associated to a point, } \\
\text { MSpi*vP } \\
\text { and defining the image of a point. } \\
\mathrm{P}^{\prime \prime}-(0,0,0)+\mathrm{MSpi}{ }^{*} \mathrm{v}\end{array}$ \\
\hline $\mathscr{R}_{(R, \pi)}$ & $\operatorname{IlCe} 3-\operatorname{Rotate}(\mathrm{Ce}, \mathrm{pi}, \operatorname{Ray}((0,0,0), \mathrm{R}))$ & \\
\hline
\end{tabular}

Table 1. GeoGebra commands to construct $\widetilde{I}_{(\mathcal{C}, p)}$ in $3 \mathrm{D}$ view and CAS view.

obtain the results presented in Sections 3 and 4 . Sometimes, we feel the need to use auxiliary applications and construct some macros. This was the case for the determination of the rotation matrices.

\section{A class of monohedral spherical tilings by four triangles}

The elements of $\mathfrak{I}_{\{\mathcal{C}, \rho)}$ are four congruent spherical triangles, but it should be pointed out that, for $\rho>\frac{\pi}{2}$ the tiles are not convex spherical polygons. The convex case was already described by several other authors, see for instance Brooks and Strantzen [3]. However, the non-convex case, $\left.\mathfrak{I}_{\langle\mathcal{C}, \rho)}, \rho \in\right] \frac{\pi}{2}, \pi[$ as far as we know, is not mentioned in the literature. We only find a brief reference to $\mathfrak{I}_{\langle\mathcal{C}, \widehat{A O C})}$ by Gaiane in [12], [13].

The construction of $\left.\mathfrak{I}_{\{\mathcal{C}, \rho)}, \rho \in\right] 0, \pi\left[\backslash\left\{\frac{\pi}{2}\right\}\right.$ is a family of four congruent triangles, all the vertices have the same valence surrounded by angles $(\alpha, \alpha, 2 \pi-\alpha)$, whit $\alpha(\rho)=\arccos \left(\frac{-\cos (\rho)}{\sqrt{1+\cos (\rho)^{2}}}\right)$.

The tiling $\mathfrak{I}_{\{\mathcal{C}, \arcsin \{\sqrt{6} / 3)\}}$ correspond to the tetrahedral spherical tilling. For some values of $\rho\left(\rho=0, \frac{\pi}{2}, \pi\right)$ we have spherical tilings by lunes (see Figure 2 ). Note that allowing $\rho>\pi$ would lead to some arcs of $\mathcal{C}$ crossing others, revealing other types of spherical pattern. 

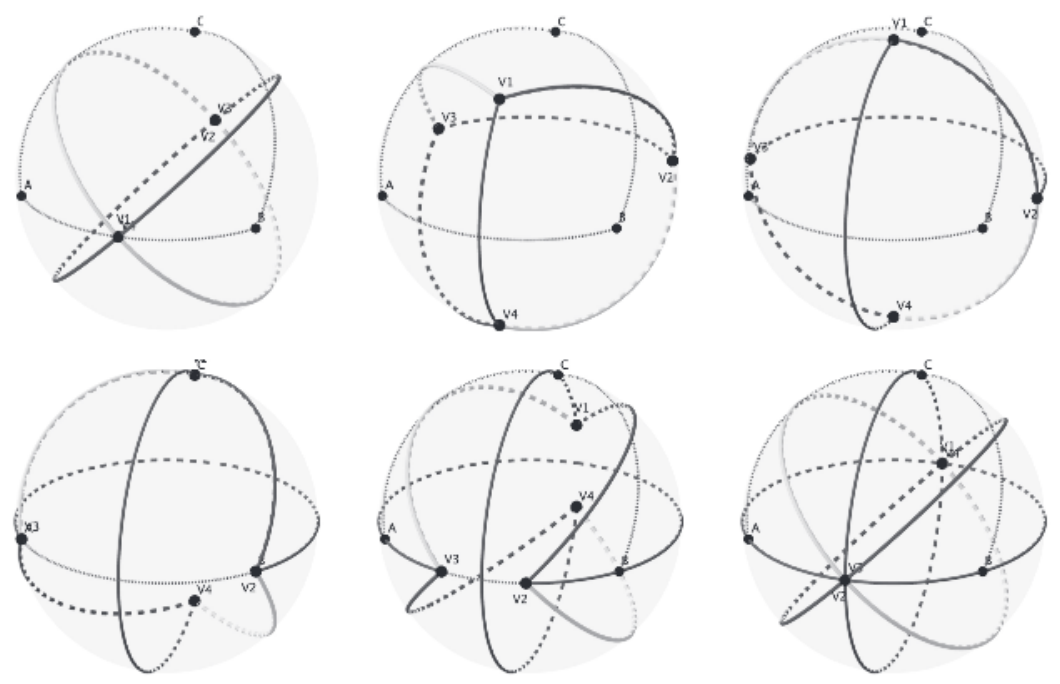

Figure 2. Representation of $\mathfrak{T}_{(C, 0)}, \mathfrak{T}_{(C, \arcsin (\sqrt{(6) / 3)})}, \mathfrak{T}_{(C, \pi / 2)}, \mathfrak{T}_{(\mathcal{C}, \widehat{A O C})}, \mathfrak{T}_{(C,(7 / 5), \widehat{A O C})}, \mathfrak{T}_{(C, \pi)}$.

\section{A class of monohedral spherical tiling by four spherical pentagons of area $\pi$}

Let us, now, present the details of the class of spherical monohedral tilings by four non-convex pentagons.

The procedure given previously applied to $\mathcal{C}$, already defined, is illustrated in Figure 3. Observe that $S$ and $S^{\prime}$ are antipodal points.

Let us summarise some of the geometric features of $\Re_{(\mathcal{C}, \rho)}=\bigcup_{i=0}^{3} \mathcal{C}_{i}$.

\begin{tabular}{|c|c|c|c|}
\hline$V_{5}$ & $V_{8}$ & $S \quad S^{\prime}$ & $V_{3} \quad S^{\prime}$ \\
\hline$b$ & $\overrightarrow{\mathcal{R}_{(S, \pi)}}$ & $\mathcal{R}_{\left(C, \frac{2 \pi}{3}\right)}$ & $\overrightarrow{\mathcal{R}_{(S, \pi)}}$ \\
\hline$V_{6}$ & & $V_{3}$ & $V_{8}$ \\
\hline Points in $\mathcal{C}$ & $\mathscr{R}_{(S, \pi)}(\mathcal{C})$ & $\mathscr{R}_{\left(C, \frac{2 \pi}{3}\right)}\left(\mathscr{R}_{(S, \pi)}(\mathcal{C})\right)$ & $\mathscr{R}_{(S, \pi)}\left(\mathscr{R}_{\left(C, \frac{2 \pi}{3}\right)}\left(\mathscr{R}_{(S, \pi)}(\mathcal{C})\right)\right)$ \\
\hline$P=V_{1}^{\rho}$ & $V_{2}^{\rho}$ & $V_{4}^{\rho}$ & $V_{7}^{\rho}$ \\
\hline$Q=V_{6}$ & $V_{3}$ & $V_{5}$ & $V_{8}$ \\
\hline$R=V_{5}$ & $V_{8}$ & $S^{\prime}$ & $S^{\prime}$ \\
\hline$S$ & $S$ & $V 3$ & $V_{6}$ \\
\hline
\end{tabular}

Figure 3. Geometric features of $\mathfrak{P}_{(\mathcal{C}, \rho)}$ 
Since we know how $\mathfrak{B}_{\{\mathcal{C}, p)}$ was built we may determine the coordinates of all its vertices. In fact,

$$
\begin{aligned}
& P\left(\frac{\sqrt{3}}{3} \cos (\rho), \frac{\sqrt{6}}{3} \cos (\rho), \sin (\rho)\right), \quad Q\left(\frac{\sqrt{3}}{3}, \frac{\sqrt{6}}{3}, 0\right), \\
& R\left(\frac{\sqrt{3}}{3},-\frac{\sqrt{6}}{6}, \frac{\sqrt{2}}{2}\right), \quad S\left(-\frac{\sqrt{3}}{3}, \frac{\sqrt{6}}{6}, \frac{\sqrt{2}}{2}\right) .
\end{aligned}
$$

The $\mathfrak{\Im}_{2}$ isometries, namely, $\mathscr{R}_{(S, \pi)}$ and $\mathscr{R}_{(C, 2 \pi / 3)}$, may be defined, respectively, by the matrices:

$$
\mathcal{R}_{1}=\left(\begin{array}{ccc}
-\frac{1}{3} & -\frac{\sqrt{2}}{3} & -\frac{\sqrt{6}}{3} \\
-\frac{\sqrt{2}}{3} & -\frac{2}{3} & 0 \\
-\frac{\sqrt{6}}{3} & \frac{1}{\sqrt{3}} & 0
\end{array}\right), \quad \mathcal{R}_{2}=\left(\begin{array}{ccc}
-\frac{1}{3} & -\frac{\sqrt{2}}{3} & -\frac{\sqrt{6}}{3} \\
\frac{2 \sqrt{2}}{3} & -\frac{1}{6} & -\frac{\sqrt{3}}{6} \\
0 & -\frac{\sqrt{3}}{2} & \frac{1}{2}
\end{array}\right)
$$

Consequently, the coordinates of the vertices of $\mathfrak{B}_{\{\mathcal{C}, \rho)}$ are:

a) for the ones depending on $\rho$ :

$$
\begin{aligned}
& V_{2}^{\rho}=\left(-\frac{\sqrt{3}}{3} \cos (\rho)-\frac{\sqrt{6}}{3} \sin (\rho),-\frac{\sqrt{6}}{3} \cos (\rho)+\frac{\sqrt{3}}{3} \sin (\rho), 0\right) \\
& V_{4}^{\rho}=\left(\frac{\sqrt{3}}{3} \cos (\rho),-\frac{\sqrt{6}}{6} \cos (\rho)-\frac{\sqrt{3}}{2} \sin (\rho), \frac{\sqrt{2}}{2} \cos (\rho)-\frac{1}{2} \sin (\rho)\right), \\
& V_{7}^{\rho}=\left(-\frac{\sqrt{3}}{3} \cos (\rho)+\frac{\sqrt{6}}{3} \sin (\rho), \frac{\sqrt{6}}{6} \cos (\rho)+\frac{\sqrt{3}}{6} \sin (\rho),-\frac{\sqrt{2}}{2} \cos (\rho)-\frac{1}{2} \sin (\rho)\right) ;
\end{aligned}
$$

b) for the others:

$$
\begin{aligned}
& V_{3}=\left(-\frac{\sqrt{3}}{3},-\sqrt{\frac{2}{3}}, 0\right) \\
& V_{8}=\left(-\frac{\sqrt{3}}{3}, \frac{\sqrt{6}}{6},-\frac{\sqrt{2}}{2}\right) .
\end{aligned}
$$

A planar representation of the tiling $\mathfrak{P}_{\{\mathcal{C}, p)}$, is shown in Figure 4. Accordingly, we have,

$$
2 \alpha_{1}+\alpha_{2}=2 \pi \quad \text { and } \quad \alpha_{3}+\alpha_{4}=2 \pi
$$

where $\alpha_{1}(\rho)=\arccos \left(\frac{-\cos (\rho)}{\sqrt{1+\cos (\rho)^{2}}}\right), \alpha_{3}(\rho)=\frac{3 \pi}{4}+\arccos \left(\frac{\sqrt{2} \cos (\rho)}{\sqrt{1+\cos (\rho)^{2}}}\right)$. 


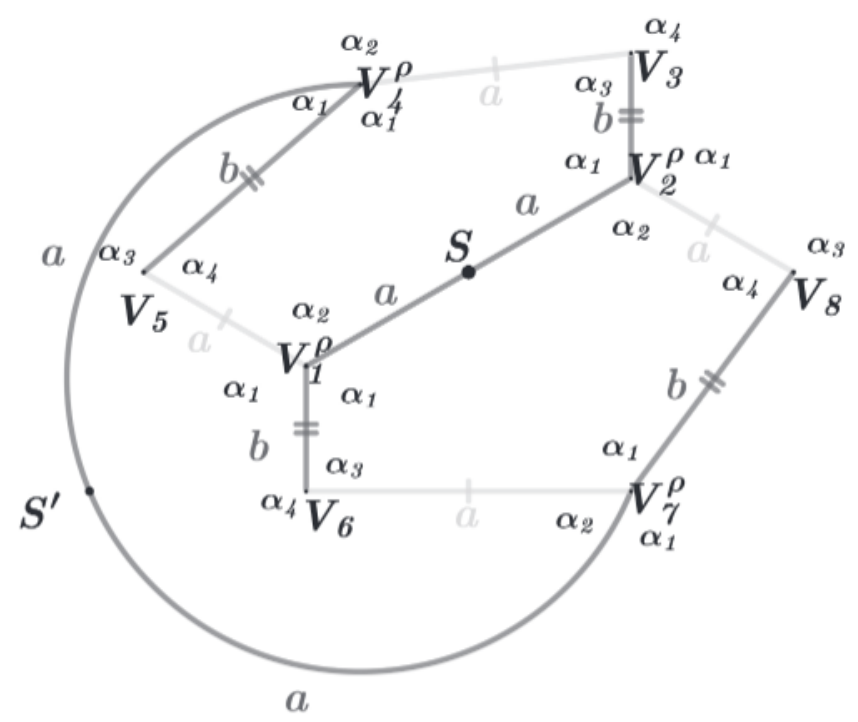

Figure 4. Planar representation of tiling $\mathfrak{P}_{(\mathcal{C}, \rho)}$

The obtained configuration, see Figure 4, defines a monohedral tiling of the sphere by four non convex pentagons if the points $V_{4}^{\rho}, S^{\prime}, V_{7}^{\rho}$ belong to a same great circle. Obverve that the isometry $\mathscr{R}_{(S, \pi)}$ sends the point $S$, corresponding to the midpoint of $\widehat{V_{1}^{\rho}} V_{2}^{\rho}$, to itself. Besides, $\overrightarrow{V_{4}^{\rho} S^{\prime}} \otimes \overrightarrow{S^{\prime} V_{7}^{\rho}}=\left(\frac{\sqrt{3}}{3} \cos (\rho)+\right.$ $\left.\left.\left.\frac{\sqrt{6}}{6} \sin (\rho), \frac{\sqrt{6}}{3} \cos (\rho)\right)-\frac{\sqrt{3}}{6} \sin (\rho)\right), \frac{1}{2} \sin (\rho)\right)$, and so, we may conclude that $V_{4}^{\rho} V_{7}^{\rho}$ is an edge of the tiling $\mathfrak{B}_{(\mathcal{C}, \rho)}$.

The points $V_{i}, i \in\{1, \ldots, 8\}$, are then vertices of four non-convex congruent spherical pentagons, each one of area $\pi$, whose length edges are $(b, a, 2 a, b, a)$ with $b=\rho$ and $a=\arccos \left(\frac{\sqrt{2}}{2} \sin (\rho)\right)$, see Table 2 .

The case of $\mathfrak{B}_{(\mathcal{C}, \rho)}$ with $\rho=\arccos \left(-\frac{1}{\sqrt{3}}\right)$ corresponds to the tetrahedral spherical tiling and the cases corresponding to $\rho \in\{0, \pi\}$ are lunes.

For each $\rho \in] 0, \pi\left[\backslash\left\{\frac{1}{2} \arccos \left(-\frac{1}{3}\right)\right\}, \mathfrak{B}_{(\mathcal{C}, \rho)}\right.$ is a monohedral tiling wih four non-convex pentagonal faces and eight vertices, six of them of valence 3 surrounded by angles $\left(\alpha_{1}, \alpha_{1}, \alpha_{2}\right)$ being the other two of valence 2 , surrounded by angles $\left(\alpha_{3}, \alpha_{4}\right)$.

If $\rho=\arccos \left(-\frac{1}{\sqrt{3}}\right)$ the tiling $\mathfrak{B}_{(\mathcal{C}, \rho)}$ defines a known dihedral tiling of the sphere by eight spherical right triangles.

\section{Conclusions and future works}

In this work, we present classes of monohedral tilings of the sphere obtained with the aid of GeoGebra. The use of special tools created in GeoGebra, for the study 


\begin{tabular}{|c|c|c|c|}
\hline$T_{1}$ & $T_{2}$ & $T_{3}$ & $T_{4}$ \\
\hline$\widehat{V_{1}^{\rho}} V_{2}^{\rho}-2 a$ & $\widehat{V_{1}^{\rho}} V_{2}^{\rho}-2 a$ & $\widehat{V} \hat{P}_{2}-b$ & $\widehat{V_{1}^{\rho}} V_{6}-b$ \\
\hline$V_{2}^{\rho} V_{3}-b$ & $V_{2}^{\rho} V_{8}-a$ & $\widehat{V_{3} V_{4}^{\rho}}-a$ & $\widetilde{V_{6} V_{7}^{\rho}}-a$ \\
\hline$\widetilde{V_{3} V_{4}^{\rho}}-a$ & $\widetilde{V_{8} V_{7}^{\rho}}-b$ & $\widehat{V_{4}^{\rho} V_{7}^{\rho}-2 a}$ & $\widehat{V_{7}^{\rho} V_{4}^{\rho}-2 a}$ \\
\hline$\widehat{V_{4}^{\rho}} V_{5}-b$ & $\widehat{V_{7}^{\rho} V_{6}}-a$ & $\widehat{V_{7}^{\rho}} V_{8}-b$ & $V_{4}^{\rho} V_{5}-b$ \\
\hline$\widehat{V_{5} V_{1}^{p}-a}$ & $\widehat{V_{6} V_{1}^{\rho}}-b$ & $\widehat{V_{8}} V_{2}^{p}-a$ & $\widehat{V_{5} V_{1}^{\rho}-a}$ \\
\hline$V_{5} \widehat{V_{1}^{\rho}} V_{2}^{\rho}-\alpha 2$ & $V_{6} \widehat{V_{1}^{\rho}} V_{2}^{\rho}-\alpha 1$ & $\widehat{V_{8}} \widehat{V_{2}^{o}} V_{3}-\alpha 1$ & $V_{5} \widehat{V_{1}^{p}} V_{6}-\alpha 1$ \\
\hline$V_{1}^{\rho} V_{2}^{\rho} V_{3}-\alpha 1$ & $V_{1}^{\rho} V_{2}^{\rho} V_{8}-\alpha 2$ & $V_{2}^{\rho} V_{3} V_{4}^{\rho}-\alpha 4$ & $V_{1}^{\rho} V_{6} V_{7}^{\rho}-\alpha 4$ \\
\hline$V_{2}^{\rho} V 3 V 4-\alpha 3$ & $V_{2}^{\rho} V_{8} V_{7}^{\rho}-\alpha 4$ & $V_{3} V_{4}^{p} V_{7}^{p}-\propto 2$ & $V_{6} V_{7}^{\rho} V_{4}^{\rho}-\alpha 2$ \\
\hline$\sqrt{V_{3}^{\rho}} V_{5}-\alpha 1$ & $\sqrt{V_{8}^{p}} V_{6}-\alpha 1$ & $V_{4}^{\rho} V_{7}^{\rho} V_{\mathrm{B}}-\alpha 1$ & $V_{7}^{\bar{\rho} V_{4}^{\rho}} V_{5}-\alpha 1$ \\
\hline$V_{4}^{\rho} V_{5} V_{1}^{\rho}-\alpha 4$ & $V_{7}^{\rho} V_{6} V_{1}^{\rho}-\alpha 3$ & $V_{7}^{\rho} V_{8} V_{2}^{\rho}-\alpha 3$ & $V_{4}^{\widehat{\rho} V_{5}} V_{1}^{\rho}-\alpha 3$ \\
\hline
\end{tabular}

Table 2. Edge and angle measures of $\mathfrak{P}_{(\mathcal{C}, \rho)}$
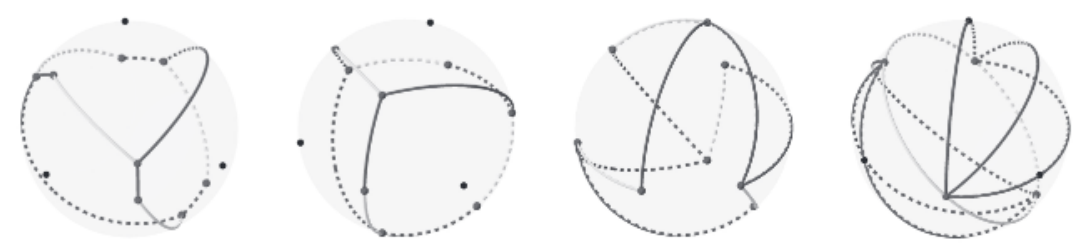

Figure 5. (a) $\mathfrak{P}_{(\mathcal{C}, \pi / \mathbb{B})},\left\{\right.$ b) $\mathfrak{P}_{(1 / 2 \arccos (-1 / 3))},\left(\right.$ c) $\mathfrak{P}_{(\mathcal{C} \text {, arcos }(-1 / \sqrt{3}))}$, (d) $\mathfrak{P}_{(\mathcal{C}, \pi)}$.

of spherical tilings, have proved to be quite useful for the search of new ones. In future works we intend to generalise the strategy described here, to be apllied to more generic cells, hoping to give a contribution to the current knowledge on this subject.

Acknowledgments. This work was supported in part by the Portuguese Foundation for Science and Technology (FCT Fundação para a Ciência e a Tecnologia), through CIDMA Centre for Research and Development in Mathematics and Applications, within project UID/MAT/04106/2013.

\section{References}

[1] D. Austin and W. Dickinson. "Spherical easel. A spherical drawing program." (2009).

[2] A. M. Breda, "A class of tilings of S2." Geometriae Dedicata 44:3 (1992), 241-253.

[3] J. Brooks and J. Strantzen, "Spherical triangles of area $\pi$ and isosceles tetrahedra." Mathematics Magazine 78:4 (2005), 311-314. 
[4] H. L. Davies, "Packings of spherical triangles and tetrahedra." Proc. Colloquium on Convexity (Copenhagen, 1965). 1965.

[5] R. J. Dawson, "Some New Tilings of the Sphere with Congruent Triangles." Renaissance Banff: Mathematics, Music, Art, Culture. Bridges Conference, 2005.

[6] R. J. Dawson, "Tilings of the sphere with isosceles triangles." Discrete \& Computational Geometry 30:3 (2003), 467-487.

[7] J. M. Dos Santos, and A. M. Breda. "Spherical Geometry and Spherical Tilings with GeoGebra" (2017). Manuscript submitted for publication.

[8] J. Erdős, "Sphaerica: Interactive Spherical Geometry Software." Proceedings of Bridges 2010: Mathematics, Music, Art, Architecture, Culture. Tessellations Publishing, 2010 .

[9] H. Gao, N. Shi, and M. Yan, "Spherical tiling by 12 congruent pentagons." Journal of Combinatorial Theory, Series A 120:4 (2013), 744-776.

[10] M. Hohenwarter and K. Fuchs. "Combination of dynamic geometry, algebra and calculus in the software system GeoGebra." Computer Algebra Systems and Dynamic Geometry Systems in Mathematics Teaching Conference. 2004.

[11] D. M. Y. Sommerville, "VI. - Division of Space by Congruent Triangles and Tetrahedra." Proceedings of the Royal Society of Edinburgh 43 \{1924), 85-116.

[12] G. Y. Panina, "Planar pseudo-triangulations, spherical pseudo-tilings and hyperbolic virtual polytopes." arXiv preprint tnath/0607171 (2006).

[13] G. Y. Panina, "Pointed spherical tilings and hyperbolic virtual polytopes." Journal of Mathematical Sciences 175:5 (2011), 591-599.

[14] Y. Ueno and Y. Agaoka, "Tilings of the 2-dimensional sphere by congruent right triangles." Memoirs of the Faculty of Integrated Arts and Sciences, Hiroshima University. IV, Science reports: studies of fundamental and environmental sciences 22 (1996), 1-23.

[15] Y. Ueno and Y. Agaoka, "Classification of tilings of the 2-dimensional sphere by congruent triangles." Hiroshima Mathematical Journal 32:3 (2002), 463-540.

Received October 25, 2017; revision received November 24, 2017

A. M. D. Breda, Departamento de Matemática, Universidade de Aveiro,

Campus Universitário de Santiago, 3810-193 Aveiro, Portugal

E-mail: ambreda@ua.pt

J. M. D. S. Dos Santos, Universidade Aberta, Rua da Escola Politécnica 141-147,

1269-001 Lisboa, Portugal

E-mail: dossantosdossantos@gmail.com 
